\title{
A Nonlinear Adaptive Level Set for Intravascular Ultrasound Images
}

\section{Segmentation}

\author{
Mehdi Eslamizadeh ${ }^{1}$, Nader Jafarnia Dabanloo ${ }^{2}$, Gholamreza Attarodi ${ }^{2}$, \\ Javid Farhadi Sedehi ${ }^{2}$, Mehrdad Mohandespoor ${ }^{2}$
}

\author{
${ }^{1}$ Biomedical Engineering Department, Qazvin Islamic Azad University, Qazvin, Iran \\ ${ }^{2}$ Biomedical Engineering Department, Science and Research Branch, Islamic Azad University, \\ Tehran, Iran
}

\begin{abstract}
In this paper, a level set method (LSM) with the aim of segmenting lumen and non-lumen pixels and Hidden Markov Random Field (HMRF) with the purpose of computing boundaries of lumen are proposed. This proposed methods was evaluated on IVUS images of 7 patients and also our results have shown that using LSM-HMRF methods leads to increasing accuracy up to $85 \%$. Results also showed that combination of LSM-HMRF could successfully identify the lumen boundary. The main advantage of this method is that one pattern using LSM from all of IVUS images is obtained. The simulation results depicted the effectiveness or the proposed method.
\end{abstract}

\section{Introduction}

In comparison with other medical imaging systems IVUS has many scientific benefits and has the ability to detect objects clearly. $\mathrm{ACC}^{1}$ and $\mathrm{AHA}^{2}$ mentioned the following phrase about angioplasty which is used with the aim of treating coronary artery diseases:

The limitation of angioplasty in detection of diseases and also the guidance of intervention processes in coronary artery could be reduced by additional technology such as intravascular ultrasound [1]. During the process of sending catheter into the vessel about hundreds or thousands of images are recorded. Thus computerized detection of middle and external vascular partitions of lumen boundaries for the investigation of blockage rate and calculation of internal space that blood flows through it is indispensable. This area in IVUS images is shown darkly and can be a sign of detection in vascular partitions [2]. In other words, since the bloody red cells inside the lumen leads to creating large scattering among ultrasound signals detection of lumen

'American College of Cardiology boundaries is more challenging especially when high frequency transducers are utilized [3]. In order to solve dissection problems different algorithms are proposed such as graph-based, gradient-based with dynamic programming, formable models with combinations of statistical features based on intensity of bloody and non-bloody areas, probability-based methods, active counter methods and multi-scale ones.

\section{The proposed algorithm for lumen detection}

At the beginning the segmentation algorithm prepares a primary guess in IVUS images with the purpose of lumen segmentation. Then, this program should cause a procedure for this curve. The output of this program is a 3-dimensional procedure which is used in main program with the aim of executing segmentation on considered image. Moreover, the zero level set counter is extracted from this procedure as final segment. The main relation is as follows, which has 3 main terms and also nonlinear and adaptive expressions can be seen in this relation:

$$
\begin{aligned}
& \frac{\partial \varphi}{\partial t}=-\mu\left[\Delta \varphi-\operatorname{div}\left(\frac{\nabla \varphi}{|\nabla \varphi|}\right)\right]- \\
& \lambda \delta(\varphi) \operatorname{div}\left(\frac{\nabla \varphi}{1+P\left(\Omega_{1} \mid I\right) P\left(\Omega_{2} \mid I\right)\left|\nabla \mathrm{G}_{\sigma}{ }^{*} I\right|^{m}|\nabla \varphi|}\right. \\
& +\frac{k \delta(\varphi)\left(\frac{1}{1+\exp (-\varsigma A d f(x, y))}-0.5\right)}{1+P\left(\Omega_{1} \mid I\right) P\left(\Omega_{2} \mid I\right)\left|\nabla \mathrm{G}_{\sigma}^{*} I\right|^{m}}
\end{aligned}
$$

${ }^{r}$ American heart association 


\subsection{Implementation of first term}

The first term in (1) depicts that $\varphi$ function should not far away from the main found segments. The second one lets the procedure to be small or big and finally the third one can be used for sending external parts out and internal parts in. The $\mathrm{k}$ is positive in external parts and is in negative parts. In order to implemet bayesian rules the prior probability is required, which depicts that how much a pixel is belonged to object and background. The prior probability for image is considered $\frac{1}{3}$ for object and $\frac{2}{3}$ for background, the first term is implemented in Matlab using Laplacian operator.

\subsection{Implementation of second term}

In order to extract zero level set procedure unit step function and impulse one are required and as a result all the calculation should be done around zero level set and procedure function should be in intersection position with zero plane. Moreover, the proposed impulse function is as follows:

$$
\delta_{\varepsilon}(x)=\frac{1}{\pi} \frac{\varepsilon^{2}}{x^{2}+\varepsilon^{2}}
$$

Where $1<\varepsilon<5$ is a constant and it should be noted that increasing this parameter leads to smoothness of the process. It is required to take a gradient from constructed procedure for IVUS and taking divergence of nominator and denominator with the purpose of implementing the second term.

\subsection{Implementation of third term}

For implementing the third term adf function should describe. It is a function that is used for creating revolution of curve procedure, which should be robust against the position of primary curve and also utilizes global statistical features. $\sigma_{1}, \mu_{1}, \sigma_{2}, \mu_{2}$ Parameters are average and standard deviation of object, which is updated in a loop that procedure function varies and new counter is calculated. These parameter are updated as follows:

$$
\begin{aligned}
& \mu_{1}= \frac{\int_{\Omega} I(x, y) H(\varphi) d x d y}{\int_{\Omega} H(\varphi) d x d y}, \\
& \mu_{2}= \frac{\int_{\Omega} I(x, y)(1-H(\varphi)) d x d y}{\int_{\Omega}(1-H(\varphi)) d x d y} \\
& \sigma_{1}= \frac{\int_{\Omega}\left(I(x, y)-\mu_{1}\right)^{2} H(\varphi) d x d y}{\int_{\Omega} H(\varphi) d x d y}, \\
& \sigma_{2}= \int_{\Omega}\left(I(x, y)-\mu_{1}\right)^{2}(1-H(\varphi) d x d y \\
& \int_{\Omega}(1-H(\varphi)) d x d y
\end{aligned}
$$

The $\mathrm{H}$ function is heveaside. This parameters are required for extracting adf and also in this study they extracted using bayesian rule.

$$
\begin{aligned}
& A d f(x, y)=\frac{\left(I(x, y)-\mu_{1}\right)^{2}}{2 \sigma_{1}^{2}} \\
& -\frac{\left(I(x, y)-\mu_{2}\right)^{2}}{2 \sigma_{2}^{2}}-\ln \frac{\sigma_{1}}{\sigma_{2}}
\end{aligned}
$$

This function suggests a new regulation for revolution procedure of IVUS. Considering the revolution curve has a disadvantage that when its value is high weak boundaries are subject to lose and when its value is small convergence speed reduces. In order to overcome these difficulties and speeding the process up an equilibrium between these two states is introduced, which is as follows:

$v(x, y)=k\left[\begin{array}{l}\frac{1}{1+\exp (-\varsigma A d f(x, y))} \\ -0.5\end{array}\right]$

Where $P\left(\Omega_{1} \mid I\right)$ is the approximate probability, which considers if a pixel belongs to object or not, $T_{p}$ is the threshold that assumed to be 0.5 and $k, \zeta$ are constant parameters that control magnitude and the non-linear degree of speed. Whenever the Adf $(x, y)$ is positive then the closed curvature will 
expand so that it will include $(x, y)$ pixel the reason why is that this pixel belongs to object. In other words. Closed curvature should reduce so that it won't include a pixel which is belonged to background. The above relation is a sigmoid function that transfers around vertical axes, this procedure is shown in figure 2. Whenever $P\left(\Omega_{1} \mid I\right)$ is near to $T_{p}$ pixels are similar to object and background and as a results belong to boundaries.

$$
P\left(\Omega_{1} \mid I\right)-\mathrm{T}_{p}
$$

The $k$ parameter is equal to 15 and $\varsigma$ is equal to 2 .

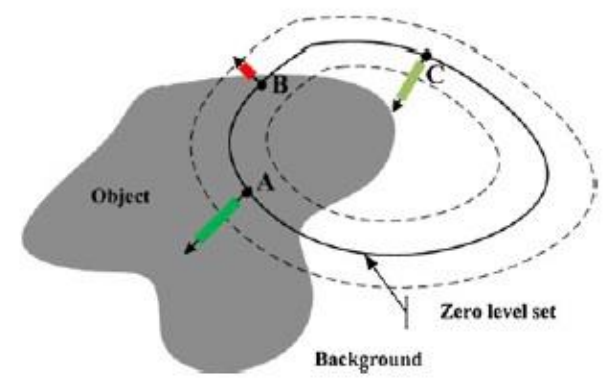

Figure 1: The revolution of curvature using nonlinear adaptive speed.

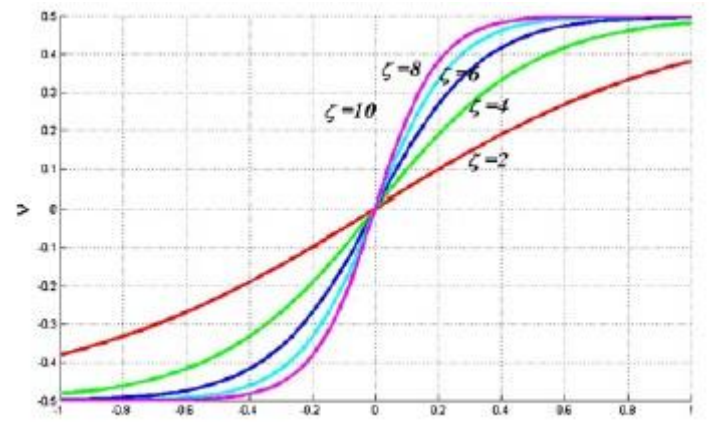

Figure 2: Sigmoid function using different values of

After implementing the third term an adaptive relation should provide with the purpose of making new procedure and updating all the considered weights, which the following relation is utilized with this purpose. This function causes a 3-dimensional procedure based on 2-dimensinal image and neighborhood according to (7):

$$
\varphi_{0}=\left\{\begin{array}{cc}
d & (x, y) \in \Omega_{0}-\sigma \Omega_{0} \\
0 & (x, y) \in \sigma \Omega_{0} \\
-d & (x, y) \in \Omega-\Omega_{0}
\end{array}\right\}
$$

Where $\mathrm{d}$ is a constant, $\Omega$ is the magnitude of image, $\Omega_{0}$ is a closed area with a primary closed curvature $\sigma \Omega_{0} . \lambda$ is a constant and the bigger is the more smooth is the curvature. For writing probable rules of bayesian (8) and (9) relations are implemented. Moreover, the prior probability is $\frac{1}{3}$, which reflects the probability of a pixel belongs to object.

$$
\begin{aligned}
& P\left(I(x, y) \mid \Omega_{i}\right)= \\
& \frac{1}{2 \pi \sigma} \exp \left(\frac{\left(I(x, y)-\mu_{i}\right)^{2}}{2 \sigma_{i}^{2}}\right) \\
& P\left(\Omega_{1} \mid I\right)= \\
& \frac{P\left(\mathrm{I} \mid \Omega_{1}\right) P\left(\Omega_{1}\right)}{P\left(\mathrm{I} \mid \Omega_{1}\right) P\left(\Omega_{1}\right)+P\left(\mathrm{I} \mid \Omega_{2}\right) P\left(\Omega_{2}\right)}
\end{aligned}
$$

\section{Discussion and Conclusion}

Utilized data base in this study is extracted from BSD-300. The proposed algorithm has some geometrical shapes and using execution of this algorithms on images the obtained results are investigated.

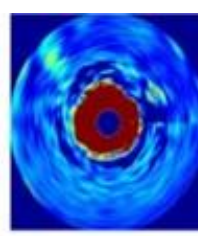

A

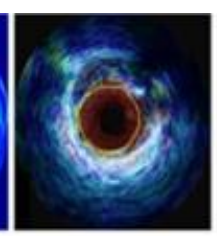

B

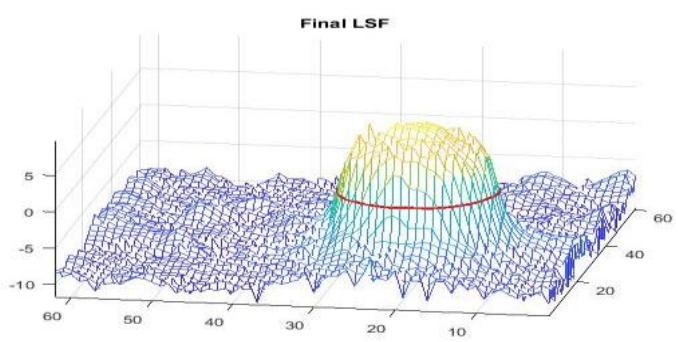

Figure 3: (A) The created map from blood, (B) the fusion of blood map with grayscale image of IVUS and $(\mathrm{C})$ manual extraction of lumen boundary (yellow color ), computerized extraction lumen boundary (red color ) after neural network thresholding 
As can be seen the claim that initial condition of images changes its situation is accepted and a rectangle is considered as initial condition and it is converted to a circle.

\section{References}

[1] B. Wang, X. Gao, D. Tao and X. Li, "A nonlinear adaptive level set for image segmentation", IEEE Transactions on Cybernetics, 44(3), 418-428, 2014.

[2] M. Eslamizadeh, G. Attarodi, N. Jafarnia Dabanloo, J. Farhadi Sedehi and S. K. Setaredan, "The segmentation of lumen boundaries at intravascular ultrasound images using fuzzy approach", Computing, 44, 1, 2017.

[3] A. Katouzian, B. Baseri, E. E. Konofagou and A. F. Laine, "Automatic detection of blood versus nonblood regions on intravascular ultrasound (IVUS) images using wavelet packet signatures", Ultrasonic Imaging and Signal Processing, 2008.

Address for correspondence:

Mehdi Eslamizadeh at Biomedical Engineering

Department, Qazvin Islamic Azad University, Qazvin, Iran

m_eslamizadeh_2006@yahoo.com

Nader Jafarnia Dabanloo at Biomedical Engineering

Department, Science and Research Branch, Islamic Azad

University, Tehran, Iran

n_jafarnia@yahoo.com

Gholamreza Attarodi at Biomedical Engineering Department, Science and Research Branch, Islamic Azad University, Tehran, Iran

attarodi@gmail.com 